\title{
A novel intelligent instrument for the detection and monitoring of thrust reverse noise at airports
}

\author{
C. Asensio ${ }^{1}$, M. Ruiz $^{1}$, M. Recuero ${ }^{1}$, G. Moschioni ${ }^{2}$, M. Tarabini ${ }^{2}$ \\ ${ }^{1}$ Universidad Politécnica de Madrid (I2A2), 28031 Madrid, ETSI Topografía-Ctra. de Valencia km 7, Spain \\ ${ }^{2}$ Politecnico di Milano (Dipartimento di Meccanica), 23900 Lecco, Via d'Oggiono 18, Italy
}

\begin{abstract}
Many airports all over the world have established restrictions for the use of thrust reverse for slowing down aircraft after landings, especially during the night period, as a way of reducing noise impact and the number of complaints in the vicinity of airports. This is the case of Madrid airport, where the Universidad Politécnica de Madrid, in collaboration with AENA, and the Politecnico di Milano have been researching, and developing intelligent instruments to improve the detection and classification of thrust reverse noise among other noise sources present in the airport. Based on a traditional approach, the thrust reverse noise detection tool detects two consecutive sound events, and applies pattern recognition techniques for the classification of each of them (such as landing and thrust reverse). A second improvement refers to the use of a microphone array linked to a noisemonitoring unit, which enables tracking the direction of arrival of the sound, thus improving the classification rates. By taking the latter, it is also possible to track the aircraft location along the runway, which enables sound pressure measurements to be transformed into sound power level estimations. Although the novel instrument can still be optimized and customized, the results have shown quite good classification rates (over $90 \%$ ).
\end{abstract}

\section{Section: RESEARCH PAPER}

Keywords: Airport noise; thrust reverse; aircraft; noise regulations, detection, classification

Citation: C. Asensio, M. Ruiz, M. Recuero, G. Moschioni, M. Tarabini, A novel intelligent instrument for the detection and monitoring of thrust reverse noise at airports, Acta IMEKO, vol. 4, no. 1, article 3, February 2015, identifier: IMEKO-ACTA-04 (2015)-01-03

Editor: Paolo Carbone, University of Perugia

Received October $24^{\text {th }}, 2013$; In final form April 21 ${ }^{\text {th }}, 2014$; Published February 2015

Copyright: @ 2014 IMEKO. This is an open-access article distributed under the terms of the Creative Commons Attribution 3.0 License, which permits unrestricted use, distribution, and reproduction in any medium, provided the original author and source are credited

Funding: This work was supported by 12A2 research group of the Universidad Politécnica de Madrid

Corresponding author: César Asensio, e-mail: casensio@i2a2.upm.es

\section{INTRODUCTION}

Noise is a major reason for concern regarding environmental protection around airports. Among all the noise sources, the activation of the thrust reverser to slow down the aircraft after landing is well known by airport authorities as a major cause of acoustic impact (and also emissions), annoyance and complaints in the vicinity of airports. Although the weight of this noise source on the overall long-term average noise assessment can be moderate (for instance in terms of day-evening-night and night-time

\section{Duration / strength / separation}
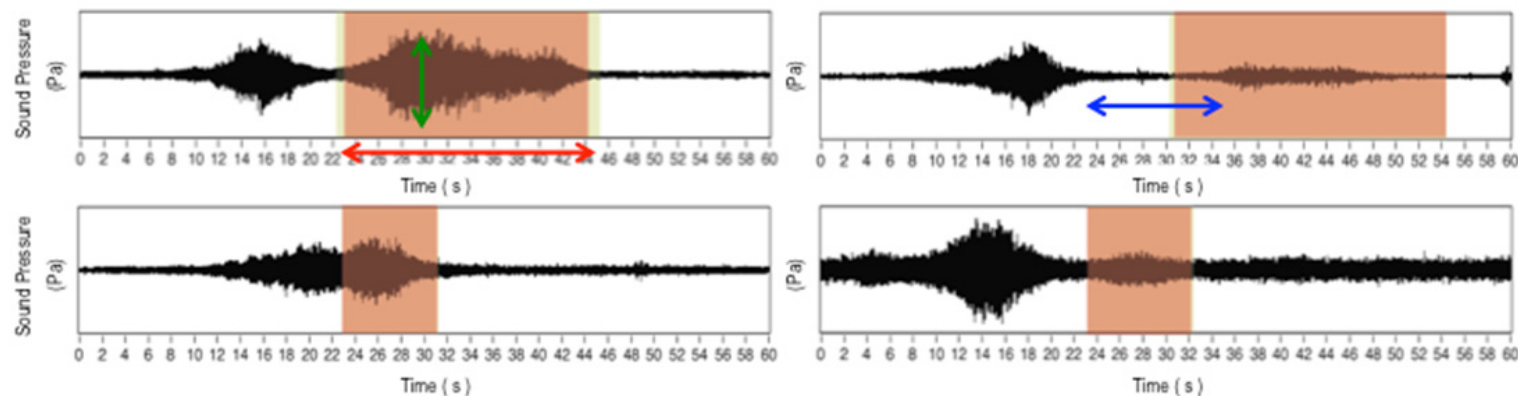

Time (s)

Figure 1. Examples of audio signals of a landing with thrust reverse activation (highlighted). 


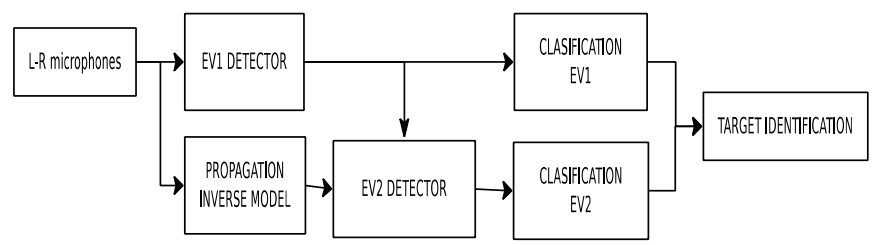

Figure 2. Thrust reverse noise detection system block diagram.

noise indicators for strategic noise mapping purposes), especially in those airports where runways are used either for landings and take-offs, the reverse thrust can be quite disturbing, as a rapid change of engine power from idle to reverse occurs causing a sudden burst of noise [1-4], as shown in Figure 1. Therefore, many airports have established restrictions for the use of thrust reversal after landing, especially during the night period, as a way of reducing the noise impact of airport operations on the community in circumstances where it is critical (for instance Paris-Orly, London-Heathrow, O'Hare-Chicago $[5,6])$.

The traditional approach for identifying thrust reverse noise is based on the detection of two consecutive sound events, by applying thresholds to the sound level measurements acquired by the airport's noise monitoring units. Each event is detected if the noise level is over the threshold for longer than a pre-established time period. But this technique has proved not to work properly in many cases, as there are many factors affecting the strength, separation and duration of both sound events: the aircraft model and the type of thrust reverser, the weather conditions, the company procedures, the aircraft's final destination, the pilot's behaviour... make it difficult to perform the detection task by just using a traditional threshold-based approach (Figure 1).

The poor performance of the traditional methods, in practice, disables the sanctioning procedures, reducing the efficacy of regulations in fighting noise impact, and originating misalignment between environmental and time efficiency policies. Extended and updated information regarding this specific noise source will help to keep and customize efficient thrust reverse restrictions where necessary, while removing them in airports where they are not useful (thrust reverse has many benefits regarding safety, time efficiency and maintenance cost).

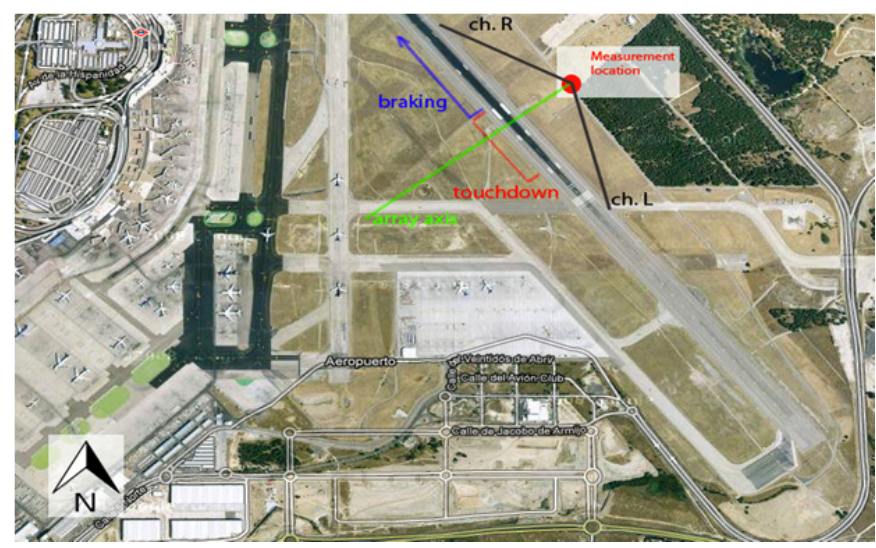

Figure 3. Field measurements scheme.

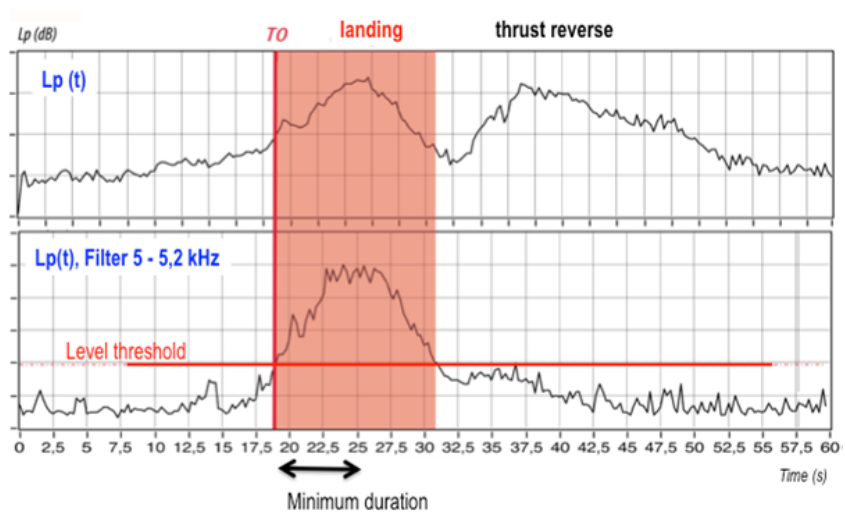

Figure 4. Example of EV1 detection.

\section{METHODOLOGY}

The methodology described in this paper is also based on a threshold detection of two consecutive sound events (EV1, for landing noise, and EV2, for thrust reverse noise). But, unlike the traditional approach, which uses the overall sound level, in this case, the detection is improved by the application of filters, acoustic modelling and signal processing practices. Furthermore, two cardioid microphones (referred to as Left and Right, L-R microphones) acquire the sound signal, making the system more robust against sounds arriving from a direction where landings are not expected.

A specific filter is applied in each of the detectors, so that the sound level is analysed for customized bandwidths. Furthermore, a transformation is applied to improve the detection of the second event (EV2), so that sound power instead of sound pressure can be analysed. If both detectors are successful, EV1 and EV2 will be classified using statistical pattern recognition techniques. In order to complete the identification of a thrust reverse sound, both sound events must be detected and properly classified. Figure 2 shows the main system block diagram and Figure 3 shows a scheme on the field locations.

\subsection{The EV1 detector}

The first stage in the TREND (thrust reverse noise detector) system is the detection of a landing sound event (EV1). A microphone acquires the sound signal, and the running sound pressure level (Lp), is calculated and used for the detection of this first sound event applying time and level thresholds. As high frequency sounds are quite

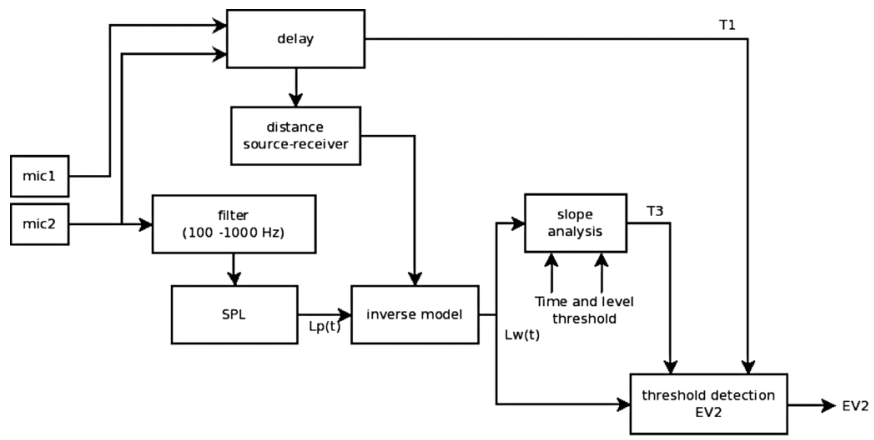

Figure 5. EV2 detector block diagram. 


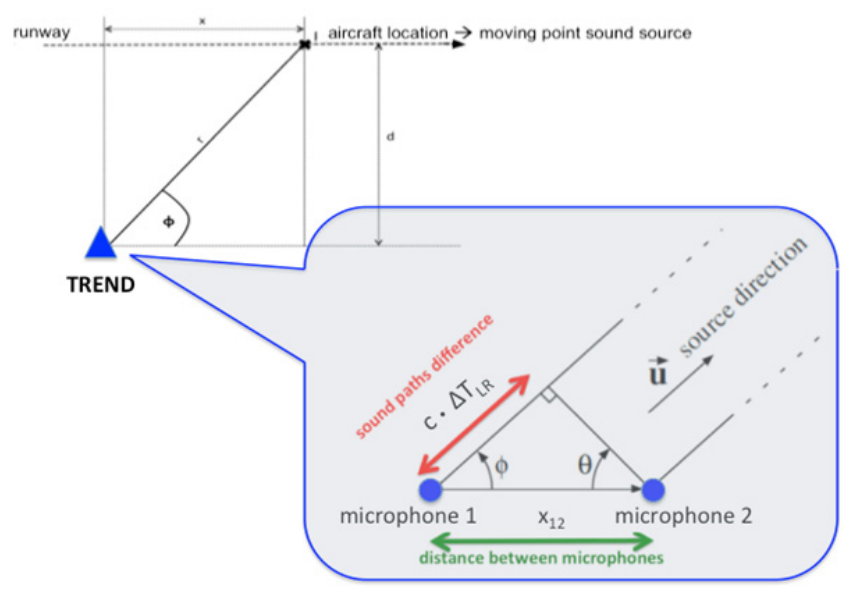

Figure 6. Aircraft location tracking.

unusual in environmental noise sources, a high frequency band pass filter ( 5.0 to $5.2 \mathrm{kHz}$ ) is applied before the Lp measurement, to improve the detection rates by reducing the false positives rates (Lp, filtered). Figure 4 shows an example of the performance of the EV1 detector.

\subsection{The EV2 detector}

When a landing (EV1) is detected, the system starts searching for EV2 (Figure 5). The EV2 detector consists of two microphones (mic1 and mic2) forming an array that is used for tracking the aircraft's position along the runway, as follows.

The delay between the signals captured in each microphone changes, depending on the relative position of the noise source (the aircraft) and the array. The delay is calculated using a cross-correlation method in the frequency domain [7]. This time delay of arrival allows estimating the direction of arrival of the sound [8], which is used to estimate the distance $(r)$ between the aircraft and the sensors (see Equation 1, where $d$ is the distance from the array to the runway, $c$ is the speed of sound, $x_{12}$ the distance between the two microphones in the array, and $\Delta T_{L R}$ is the delay obtained in the measurement). Figure 6 shows a simplified scheme of parameters involved in the source location tracking process.

$$
r=\frac{d}{\sin \left(\cos ^{-1}\left(\frac{c \cdot \Delta T_{L R}}{x_{12}}\right)\right)}
$$

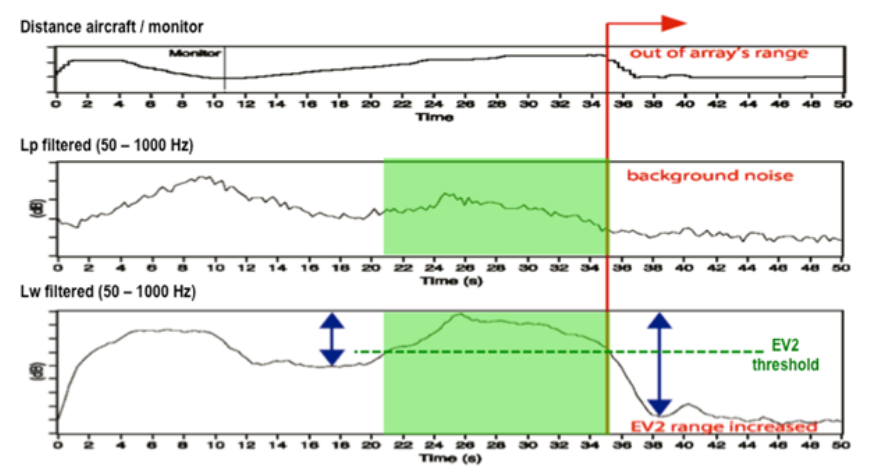

Figure 7. EV2 enhancement for detection.

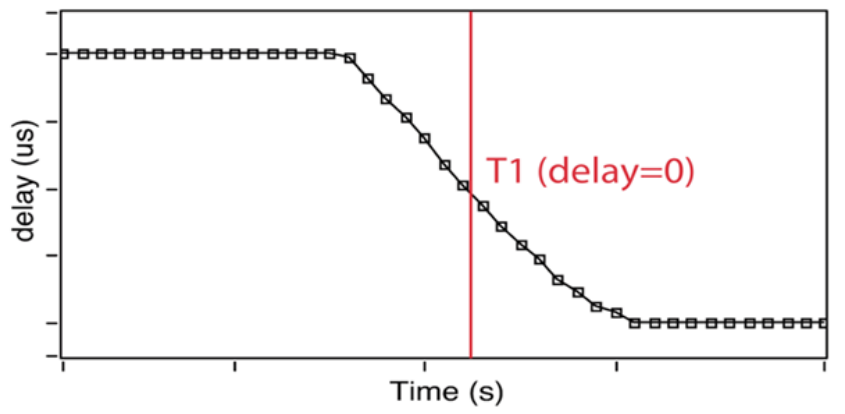

Figure 8. Time delay during a landing.

Unlike sound pressure level (SPL), the distance from the source to the receiver does not affect sound power level; therefore, when the thrust reverser is activated, the sound power emitted increases suddenly, making it easier to detect. Taking advantage of this phenomenon, and using an approach similar to the one presented by the authors in [9], the estimation of the sound power level has been carried out using a simplified inverse sound propagation model based on ISO 9613 [10], and shown in Equation 2.

$L w(t)=L p(t)+20 \log r(t)+\frac{r(t)}{1000} \alpha+A$

where $L w(t)$ is the sound power level $(\mathrm{dB}), L p(t)$ the sound pressure level $(\mathrm{dB}), r(t)$ the distance from the source to the receiver $(\mathrm{m}), \alpha$ is a coefficient describing the atmospheric attenuation of sound with the dista

nce $(\mathrm{dB} / \mathrm{km})$, and $A$ is a constant that counts for all other factors.

Using this transformation every thrust reverse sound event is enhanced, making its dynamic range higher (see Figure 7), thereby improving the performance of a threshold detector.

\subsection{EV1 detection improvement}

During landings, the aircraft arrives from the south (in this test case) and, as the distance decreases (Figure 3), the sound pressure level increases, triggering EV1 in time TO. At that time, the aircraft is at the left of the TREND system, and during EV1 the aircraft will change its position to the right. Therefore, during EV1 the array will detect a positive delay at the beginning that becomes negative in T1, which is the time when the aircraft is in front of the array axis (see Figure 8). In the event that a negative zero crossing is not perceived within EV1, the event will be rejected (this is a constraint in this measurement setup). In the event that T1 is detected, the system starts searching EV2. This is a way to reduce the EV1's false positive detection rates, as any sound event is rejected if its source was not strictly

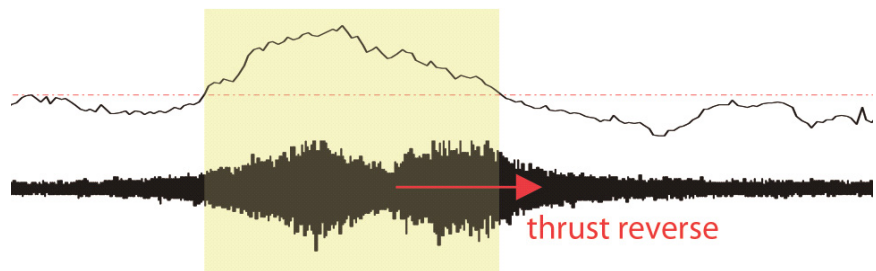

Figure 9. Performance of EV1 detector for early thrust reverse activation. 


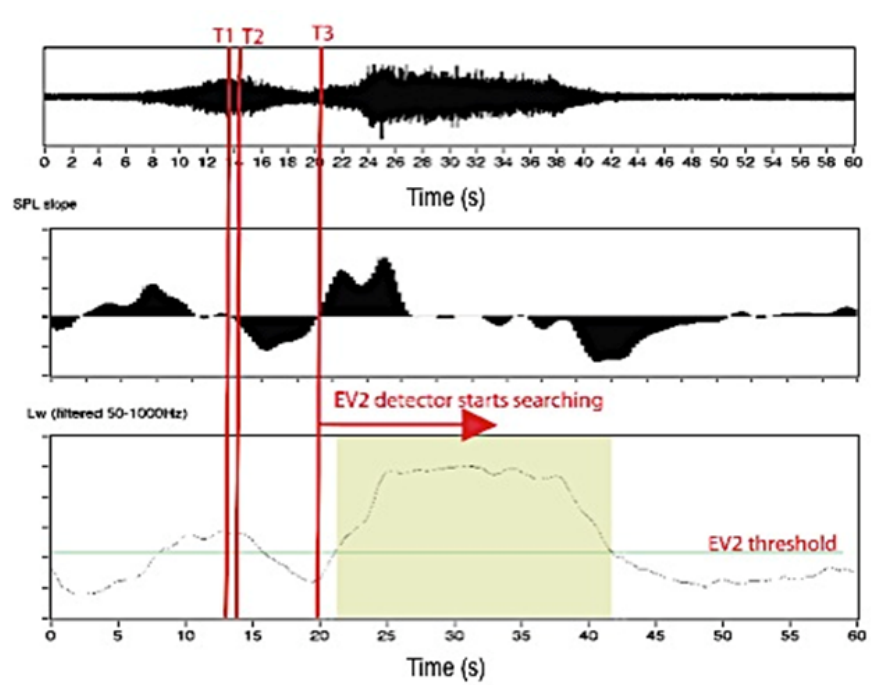

Figure 10. EV2 enhancement for detection.

moving from left to right during the detection duration.

\subsection{EV2 detection improvement}

The EV2 detection must be done once the landing sound event is finished, which should fit to the end of EV1. However, by just using a threshold-based EV1 detector, it is not possible to tune the EV1 threshold in a very accurate way for all of the cases (Figure 9). In view of those cases having "early" thrust reverse activation, the EV1 ending time cannot be a reference and there must be extra constraints to start searching for EV2.

A little time after T1 (or at T1) the estimated sound power level must start decreasing (as the aircraft is moving away slowing down). This is the time we have called T2. Only after T2, thrust reverse can be detected. This will prevent the system form false positives caused by aircraft directivity. After T2, if thrust reverse is activated, $\mathrm{Lw}(\mathrm{t})$ will increase suddenly at a high rate, determining T3. T2 and $\mathrm{T} 3$ are calculated from analysing the slope trends in the sound power level time history (by just establishing some

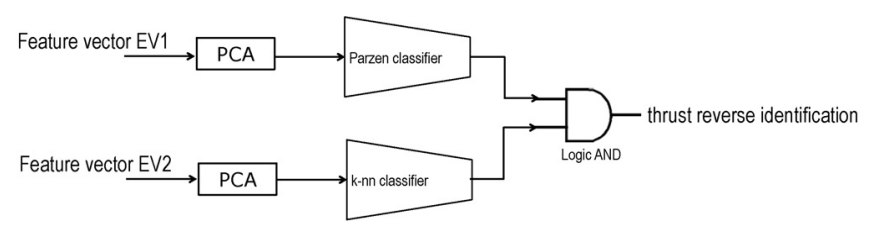

Figure 11. Thrust reverse noise identification.

thresholds for these constraints, see Figure 10). This is a way to prevent false positives caused by distant sound events in the airport (run-ups, taxi...), as thrust reverse usually produces strong level increments.

\subsection{Events classification}

After detecting two consecutive sound events (EV1 and EV2), the system classifies them independently, in order to reduce the false positives identification rates. This process is carried out through the application of statistical pattern recognition techniques.

As the classification stage is performed after an autonomous previous detection of two sound events, it was decided to use two independent classification stages for EV1 and EV2. As the characteristics of the target sounds are completely different (landings for the EV1 detector, and thrust reverse for the EV2 detector), using specific classification algorithms could improve the performance of each of the classifiers.

A feature extraction process is carried out for both events, describing the dynamic and frequency characteristics of the sounds, and also the position of the aircraft for each of the events. Mel frequency cepstral coefficients (MFCC) have shown a good performance in sound recognition applications [11-15], so the first 20 coefficients were selected. Two new features were selected to describe the evolution of the time delay between microphones during EV1 and EV2, which is highly correlated to the location and movement of the aircraft during both events.

The training and testing of the system was developed with Matlab-PRTools. The recognition process starts with a

Table 1. EV1 detection rates prior to classification

\begin{tabular}{lccc}
\hline Detection rates & EV1 Detected & EV1 not detected & Error (\%) \\
\hline Landings & 398 & 1 & 0.0 \\
Take-offs & 0 & 252 & 0.0 \\
\hline
\end{tabular}

Table 2. EV2 detection rates prior to classification

\begin{tabular}{lccc}
\hline Detection rates & EV2 Detected & EV2 not detected & Error (\%) \\
\hline Landings with thrust reverse & 315 & 14 & 4.0 \\
Landings without thrust reverse & 20 & 63 & 24.0 \\
\hline
\end{tabular}

Table 3. Overall identification results.

\begin{tabular}{lccc}
\hline Event type & Detection error rates (\%) & Classification error rates (\%) & Overall error rates (\%) \\
\hline Landing with thrust reverse & 4.0 & 4.9 & 8.5 \\
Landing without thrust reverse & 24.0 & 40.0 & 9.6 \\
\hline
\end{tabular}


principal components analysis (PCA), used to decorrelate the data. Afterwards, different algorithms were tested for each of the classifiers independently, so that those two showing the best performance were selected:

- A k-nearest neighbour [16] was used for thrust reverse events. This is a non-parametric classifier. It calculates the distance between the object to be classified and those in the training set, and assigns the class that is most repeated among the $\mathrm{K}$ nearest neighbours $(\mathrm{K}=5)$.

- A Parzen classifier [17] was used for landing events. This is a non-parametric classifier. During the training, the classifier makes a non-parametric estimation of the probability density functions of each class, which are used for the classification of new objects.

The identification of thrust reverse activation is positive if the first event is classified as landing and the second one is classified as thrust reverse noise (see Figure 11).

\section{RESULTS}

Some tests were carried out at Madrid-Barajas airport. The recordings were manually edited and labelled, creating a sound events database, consisting of 315 landings with thrust reverse activation and 83 without it.

Tables 1 and 2 show the performance of the detectors. The EV1 detector has shown a great performance: only landings are detected as EV1s, and almost every landing is detected. On the other hand, EV2 detector performance is lower, as sources other than thrust reverse are incorrectly detected as EV2. An adjustment with a higher threshold would remove many of these false positives, which are generated by aircraft taxiing or run-ups at a long distance, but, on the other hand, many thrust reverse events could also be missed. As no prior information was available regarding the number of these low level thrust reverse events, a conservative scenario was considered. However, after the classification stage, the operation point of the detectors can be optimized, and the overall identification rates will be improved.

The tests showed an error rate lower than 10\% (Table 3), which can still be optimized, on the one hand, with a proper customization of the sensors and the measurement setup, and on the other, with the use of the prior probability of thrust reverse occurrence, during the training stage of the classifier.

\section{CONCLUSIONS}

The variability in the duration, strength and separation of the landing and thrust reverse sound events cannot be properly implemented by just using thresholds on the overall running sound pressure level measurements. This is the reason why traditional threshold detectors are not effective for identifying thrust reverse noise at airports. The novelty in this paper derives from: a) The improvement in the detection through the application of signal processing to track the aircraft position and transform sound pressure into sound power levels;

b) The application of pattern recognition techniques for the classification of previously detected sound events in the process of identification thrust reverser being activated.

Obviously, aircraft will have generated most of the noise events inside an airport, making it difficult to recognize each of them, as the different sound classes are not neatly separated. Tracking the location of the aircraft along the runway optimizes the performance of both the detection and the classification stages, reducing the error rates below $10 \%$.

The sound power level estimation has shown a great performance for the detection of thrust reverse sound events, but it involves taking extra precautions, as not only the sound level is important, but also the cross-correlation between microphones (for instance, the protection of microphones against environmental conditions must be improved). It should be noted that the location of the TREND system must be carefully selected. The location must be as far as possible from the braking area in order to enhance the separation between sound events. But it must be close enough to capture the thrust reverse sound. The array parameters and the distance from the array to the runway must be chosen so that the operating angle of the instrument covers the typical landing and braking areas.

The described methodology can be implemented easily and reliably in an instrument, by simply applying common hardware and software resources to provide real time results regarding the activation of thrust reverse. These results, linked to those provided by noise monitoring units can be used, for instance, to complete any sanctioning procedure at an airport, or to analyse the effect or the need for restrictions regarding thrust reverser use. The methodology has been valid and effective for the measurements carried out at Madrid-Barajas airport [18,19].

\section{ACKNOWLEDGEMENTS}

The authors are very grateful to SEA Milano and AENA for showing their support for this project.

The authors are grateful to the Spanish "Ministerio de Educación, Cultura y Deportes" for the financial support provided to facilitate the corresponding author's research stay at the Politecnico di Milano in Italy.

\section{REFERENCES}

[1] B.M. Dunkin, A.A. Atchley, K.K. Hodgdon, Directivity and spectral characteristics of aircraft noise during landing operations, Journal of the Acoustical Society of America. 121, 2007, p. 3112.

[2] B.M. Dunkin, Directivity and spectral source noise characterization of commercial aircraft during landing with thrust reverser engagement, 2008.

[3] K.K. Hodgdon, A.A. Atchley, R.J. Bernhard, Low Frequency Noise Study, PARTNER-COE-2007-001 (2007). 
[4] R.M. Gutiérrez, A.A. Atchley, K.K. Hodgdon, Characterization of aircraft noise during thrust reverser engagement, Journal of the Acoustical Society of America. 118, 2005, p. 1852.

[5] Boeing, Airports with noise and emissions restrictions, 2012.

[6] C.C. Rice, C.M. Walton, Restricting the Use of Reverse Thrust as an Emissions Reduction Strategy. Report n ${ }^{\circ}$ SWUTC/03/167231-1 2, Report No. SWUTC/03/167231-1 2, 2001.

[7] D. Hertz, Time delay estimation by combining efficient algorithms and generalized cross-correlation methods, Acoustics, Speech and Signal Processing, IEEE Transactions on. 34, 1986, pp. 1-7.

[8] J.M. Valin, F. Michaud, J. Rouat, D. Létoumeau, Robust sound source localization using a microphone array on a mobile robot, Proceedings International Conference on Intelligent Robots and Systems, 2003.

[9] C. Asensio, I. Pavón, M. Ruiz, R. Pagan, M. Recuero, Estimation of directivity and sound power levels emitted by aircrafts during taxiing, for outdoor noise prediction purpose, Appl. Acoust. 68, 2007, pp. 1263-1279.

[10] ISO, Attenuation of sound during propagation outdoors. Part 2: General method of calculation. ISO 9613-2:199, 1996.

[11] C. Asensio, M. Ruiz, M. Recuero, Real-time aircraft noise likeness detector, Appl. Acoust. 71, 2010, pp. 539-545.
[12] A. Rabaoui, Z. Lachiri, N. Ellouze, Automatic environmental noise recognition, Industrial Technology, 2004. IEEE ICIT '04. 2004 IEEE International Conference on., vol. 3, 2004, pp. 1670-1675.

[13] A. Rabaoui, H. Kadri, N. Ellouze, New approaches based on One-Class SVMS for impulsive sounds recognition tasks, Machine Learning for Signal Processing, 2008. MLSP 2008. IEEE Workshop on., 2008, pp. 285-290.

[14] M. Cowling, R. Sitte, Comparison of techniques for environmental sound recognition, Pattern Recognition Letters, 24, 2003, pp. 2895-2907.

[15] M. Tabacchi, C. Asensio, I. Pavón, M. Recuero, J. Mir, M.C. Artal, A statistical pattern recognition approach for the classification of cooking stages. The boiling water case, Appl. Acoust. 74, 2013, pp. 1022-1032.

[16] T.M. Cover, P.E. Hart, Nearest neghbor pattern classification, IEEE transactions on information theory, 13, 1967.

[17] A.K. Jain, M.D. Ramaswami, Classifier design with Parzen windows, Pattern Recognition and artificial intelligence, 1988, pp. 221-228.

[18] C. Asensio, G. Moschioni, M. Ruiz, M. Tarabini, M. Recuero, Implementation of a thrust reverse noise detection system for airports, Transportation Research Part D: Transport and Environment, 19, 2013, pp. 42-47.

[19] C. Asensio, Aportaciones a los sistemas de discriminación de fuentes sonoras en al medida de ruido en aeropuertos, 2012. 